\title{
Collective charge-density excitations of noncircular quantum dots in a magnetic field
}

\author{
C. A. Ullrich and G. Vignale \\ Department of Physics, University of Missouri, Columbia, Missouri 65211
}

(Received 13 July 1999)

\begin{abstract}
Recent photoabsorption measurements have revealed a rich fine structure in the collective charge-density excitation spectrum of few-electron quantum dots in the presence of magnetic fields. We have performed systematic computational studies of the far-infrared density response of quantum dots, using time-dependent density-functional theory in the linear regime and treating the dots as two-dimensional disks. It turns out that the main characteristics observed in the experiment can be understood in terms of the electronic shell structure of the quantum dots. However, additional features arise if a breaking of the circular symmetry of the dots is allowed, leading to an improved description of the experimental results.
\end{abstract}

\section{INTRODUCTION}

The electronic structure and dynamics of quantum dots have been a subject of intense study in recent time. ${ }^{1,2}$ Frequently one makes the simplifying assumption that the electrons in quantum dots form a two-dimensional system confined by a parabolic potential of circular symmetry. The electronic ground state can then be found either through diagonalization schemes or by using mean-field approaches such as Hartree theory or density-functional theory. The former treatment is computationally much more demanding, and has so far only been used to describe few-electron systems, whereas the latter approach has been applied to electron numbers of order 100. In either case, however, one finds that the main features observed in the experiment (such as effects related to the electronic shell structure) are well reproduced.

The model of a parabolic circular quantum dot is of course an idealization. It was already recognized in the early 1990s that explaining certain experimental features requires including some deviation from parabolicity of the confining potential. A first study of this kind was performed by Broido et al., ${ }^{3}$ who considered the ground state and far-infrared (FIR) response of up to 30 electrons confined in a circular quantum disk of radius $100 \mathrm{~nm}$. Their approach, as well as a similar one by Gudmundsson and Gerhards, ${ }^{4}$ was based on a Hartree plus random-phase-approximation description of the electrons. Both studies showed that a correction to the confining potential toward the edge of the dot that makes it steeper than the parabolic potential leads to a blueshift of the collective dipole modes that increases with the number $N$ of electrons in the dot. In addition, it was found in Ref. 4 that one has to include a deviation from circular symmetry in order to explain the anticrossing behavior in the FIR spectra observed in the experiments by Demel et al. ${ }^{5}$ These findings were supported by an exact diagonalization study of quantum dot helium by Pfannkuche and Gerhards, ${ }^{6}$ and further elaborated by Ye and Zaremba ${ }^{7}$ using a hydrodynamic approach. We finally mention a very recent study by Hirose and Wingreen ${ }^{8}$ that used spin-density-functional theory to describe the electronic shell structure and calculate addition energies in elliptic dots.

The present work was motivated by a recent series of experiments on self-assembled InAs quantum dots performed by Kotthaus and co-workers in Munich. ${ }^{9-11}$ The experiments use a combination of in situ capacitance spectroscopy and FIR absorption spectroscopy to probe the ground state and collective charge-density excitations as a function of the electron number per dot, $1 \leqslant N \leqslant 6$. For $N=1$ and 2 one finds the well-known two-mode spectrum described by the simple formula

$$
\omega_{ \pm}=\sqrt{\omega_{0}^{2}+\frac{\omega_{c}^{2}}{4} \pm \frac{\omega_{c}}{2}}
$$

where $\omega_{0}$ is the characteristic frequency associated with a parabolic confinement potential, and $\omega_{c}=e B / m^{*} c$ is the cyclotron frequency for particles of charge $e$ and mass $m^{*}$ in a magnetic field $B$. However, for $N>2$ the authors of Refs. 9 and 10 detected a much richer FIR spectrum: the $\omega_{+}$mode is observed to split up into three subpeaks (see Fig. 3 of Ref. 9).

The authors argued within a simple picture of noninteracting particles that the observed splitting of the $\omega_{+}$mode is caused by the nonparabolicity of the confining potential of the quantum dots. Since for $N=3,4$, and 5 the $p$ shell is only partly filled, the system can perform transitions of the $s$ $\rightarrow p$ and $p \rightarrow d$ types, which have slightly different energies, in contrast with the strictly parabolic case. This simple explanation, however, can only account for a twofold splitting of the $\omega_{+}$mode. It is then further argued that the observed third (somewhat weaker) signal is caused by effects related to electron-electron interaction.

It is the purpose of the present paper to provide a theoretical explanation of the threefold splitting of the $\omega_{+}$mode observed in Ref. 9. Our approach is based on densityfunctional theory for the ground state and linear response in the presence of a magnetic field. It will turn out in the following that including electron-electron interaction effects alone is not sufficient. Instead, the presence of the third subpeak is explained by a combination of the nonparabolicity plus breaking of the circular symmetry of the confining potential.

The paper is organized as follows: in Sec. II, we shall introduce our model for the quantum dots, a two-dimensional disk, and we shall present the theoretical methods for de- 
scribing the electronic ground state and dynamics. In Sec. III, we then discuss our numerical results, and Sec. IV gives our conclusions.

\section{FORMAL FRAMEWORK}

\section{A. Ground-state calculation}

The electronic ground state of a system in the presence of magnetic fields is described by current-density-functional theory. The general formalism was developed by Vignale and Rasolt, ${ }^{12}$ and to date there exist several applications to quantum dots. ${ }^{13,14}$ Here we want to generalize the formalism, which originally was developed for circular quantum dots, to describe noncircular systems.

In the following, we shall make our lives a little easier, especially in view of the linear response calculations later on, and neglect the dependence of the exchange-correlation (xc) energy $E_{\mathrm{xc}}$ on the current density $\mathbf{j}_{p \sigma}$. This dependence is known to cause only a small effect in the electronic ground state of quantum dots. The xc vector potential then drops out, and the Kohn-Sham equation as derived in Ref. 12 becomes

$$
\begin{gathered}
\left\{-\frac{\nabla^{2}}{2 m^{*}}-\frac{i e}{2 m^{*} c}\left[\mathbf{A}_{\mathrm{ext}}(\mathbf{r}) \cdot \boldsymbol{\nabla}+\boldsymbol{\nabla} \cdot \mathbf{A}_{\mathrm{ext}}(\mathbf{r})\right]+\frac{e^{2} A_{\mathrm{ext}}^{2}(\mathbf{r})}{2 m^{*} c^{2}}\right. \\
\left.+\mathrm{v}_{\mathrm{ext} \sigma}(\mathbf{r})+\mathrm{v}_{H}(\mathbf{r})+\mathrm{v}_{\mathrm{xc} \sigma}(\mathbf{r})\right\} \psi_{j \sigma}(\mathbf{r})=\epsilon_{j \sigma} \psi_{j \sigma}(\mathbf{r}),
\end{gathered}
$$

where as usual $\mathbf{v}_{\text {ext } \sigma}$ and $\mathbf{A}_{\text {ext }}$ denote the external scalar and vector potentials and $\mathrm{v}_{H}(\mathbf{r})$ and $\mathrm{v}_{\mathrm{xc} \sigma}(\mathbf{r})$ are the Hartree and $\mathrm{xc}$ potentials. In the following, the basic assumption is that the quantum dots can be treated as two-dimensional systems. We then use polar coordinates and write the external potential as a sum of the bare potential of the quantum dot plus the Zeeman term:

$$
\mathrm{v}_{\mathrm{ext} \sigma}(r, \varphi)=\mathrm{v}_{\mathrm{dot}}(r, \varphi)+\frac{1}{2} g * \mu_{B} B \sigma
$$

Here $\mu_{B}=e \hbar / 2 m, \sigma= \pm 1$, and the specific form of $\mathrm{V}_{\mathrm{dot}}(r, \varphi)$ will be discussed in Sec. II C below. In turn, the external vector potential is given by

$$
\mathbf{A}_{\mathrm{ext}}(r, \varphi)=\frac{B}{2} r \hat{e}_{\varphi}
$$

corresponding to a uniform magnetic field $B$ perpendicular to the dot. Next we expand the Kohn-Sham orbitals in polar coordinates as

$$
\psi_{j \sigma}(\mathbf{r})=\sum_{l} \phi_{j l \sigma}(r) e^{-i l \varphi}
$$

where the $\phi_{j l \sigma}(r)$ are real. The spin density is then given by

$$
\begin{aligned}
n_{\sigma}(\mathbf{r}) & =n_{\sigma}(r, \varphi) \\
& =\sum_{j} f_{j \sigma}\left|\psi_{j \sigma}(\mathbf{r})\right|^{2} \\
& =\sum_{j l l^{\prime}} f_{j \sigma} \phi_{j l \sigma}(r) \phi_{j l^{\prime} \sigma}(r) e^{i\left(l-l^{\prime}\right) \varphi},
\end{aligned}
$$

where $f_{j \sigma}$ denotes occupation numbers of the orbitals. In the following, all calculations will be performed at a small but finite temperature, $T=4.2 \mathrm{~K}$, in order to avoid convergence problems at small magnetic fields. The occupation numbers are then given by the thermal distribution

$$
f_{j \sigma}=\frac{1}{1+\exp \left(\frac{\epsilon_{j \sigma}-\mu}{k_{B} T}\right)},
$$

and the chemical potential $\mu$ is fixed through the relation $\sum_{j \sigma} f_{j \sigma}=N$. Next the confining potential of the dot is expanded as

$$
\mathrm{v}_{\mathrm{dot}}(r, \varphi)=\sum_{l} \mathrm{v}_{\mathrm{dot}, l}(r) e^{-i l \varphi}
$$

Similar expansions are made for the Hartree and xc potentials. For the dot potential, the angular components

$$
\mathrm{V}_{\mathrm{dot}, l}(r)=\frac{1}{2 \pi} \int_{0}^{2 \pi} d \varphi e^{i l \varphi} \mathrm{V}_{\mathrm{dot}}(r, \varphi)
$$

in general have to be obtained through straightforward numerical integration, and similarly for the xc potential (for the latter we use the local-density approximation in the parametrization of Tanatar and Ceperley ${ }^{15}$ ). For the Hartree potential, one finds

$$
\begin{aligned}
\mathrm{v}_{H, l}(r)= & 2 \pi e^{* 2} \sum_{\sigma} \sum_{\substack{j n n^{\prime} \\
n-n^{\prime}=l}} \\
& \times \int_{0}^{\infty} r^{\prime} d r^{\prime} \phi_{j n \sigma}\left(r^{\prime}\right) \phi_{j n^{\prime} \sigma}\left(r^{\prime}\right) I_{l}\left(r, r^{\prime}\right),
\end{aligned}
$$

where $e^{*}$ is the effective electronic charge, and the $I_{l}\left(r, r^{\prime}\right)$ involve integrals over Bessel functions:

$$
I_{l}\left(r, r^{\prime}\right)=\int_{0}^{\infty} d q J_{l}(q r) J_{l}\left(q r^{\prime}\right) .
$$

$I_{l}\left(r, r^{\prime}\right)$ can be expressed in terms of hypergeometric functions that can be further reduced to complete elliptic integrals (see Ref. 3 for the case $l=1$ ). Inserting everything into the Kohn-Sham equation, we finally obtain

$$
\begin{aligned}
\{- & \frac{1}{2 m^{*}}\left(\frac{d^{2}}{d r^{2}}+\frac{1}{r} \frac{d}{d r}-\frac{l^{2}}{r^{2}}\right)-\frac{e l B}{2 m^{*} c}+\frac{e^{2} B^{2} r^{2}}{8 m^{*} c^{2}} \\
& \left.+\frac{1}{2} g^{*} \mu_{B} B \sigma\right\} \phi_{j l \sigma}(r)+\sum_{k}\left[\mathrm{v}_{\mathrm{dot}, k}(r)+\mathrm{v}_{H, k}(r)\right. \\
& \left.+\mathrm{v}_{\mathrm{xc} \sigma, k}(r)\right] \phi_{j l-k \sigma}(r)=\epsilon_{j \sigma} \phi_{j l \sigma}(r) .
\end{aligned}
$$


Equation (12) couples the angular components of the KohnSham orbitals (5). In practice, we of course limit the expansions of the orbitals and potentials to a finite number of components, $-L, \ldots, l, \ldots, L$, where $L$ of the order 5 is sufficient to give convergence for the cases under study. Solution of the Kohn-Sham equation (12) is then accomplished by discretization on a logarithmic radial mesh with $N_{\text {grid }}$ $\sim 100$ grid points.

\section{B. Linear response}

The FIR absorption spectra as measured in Refs. 9 and 10 are proportional to the photoabsorption cross section

$$
\sigma(\omega)=\frac{4 \pi \omega}{c} \operatorname{Im} \alpha(\omega)
$$

where the dipole polarizability with respect to, say, the $x$ axis,

$$
\alpha(\omega)=-\frac{2 e}{E_{0}} \int d^{3} r x n_{1}(\mathbf{r}, \omega),
$$

is obtained from the linear density response $n_{1}(\mathbf{r}, \omega)$ of the quantum dots to an external field of the form

$$
\mathbf{v}_{1}(\mathbf{r}, \omega)=\frac{e E_{0} x}{2}
$$

where $E_{0}$ is the amplitude of the electric field strength. In order to calculate $n_{1}(\mathbf{r}, \omega)=\Sigma_{\sigma} n_{1 \sigma}(\mathbf{r}, \omega)$, we have to solve the linear spin-density response equation, which reads as follows:

$$
\begin{aligned}
n_{1 \sigma}(\mathbf{r}, \omega)= & \int d^{3} r^{\prime} \chi_{\sigma}\left(\mathbf{r}, \mathbf{r}^{\prime}, \omega\right)\left\{\mathrm{v}_{1 \sigma}\left(\mathbf{r}^{\prime}, \omega\right)\right. \\
& +\sum_{\tau} \int d^{3} r^{\prime \prime}\left(\frac{e^{* 2}}{\left|\mathbf{r}^{\prime}-\mathbf{r}^{\prime \prime}\right|}+f_{\mathrm{xc} \sigma \tau}\left(\mathbf{r}^{\prime}, \mathbf{r}^{\prime \prime}, \omega\right)\right) \\
& \left.\times n_{1 \tau}\left(\mathbf{r}^{\prime \prime}, \omega\right)\right\},
\end{aligned}
$$

i.e., we have to solve two coupled integral equations for $n_{1 \uparrow}$ and $n_{1 \downarrow}$. The Kohn-Sham response function

$$
\chi_{\sigma}\left(\mathbf{r}, \mathbf{r}^{\prime}, \omega\right)=\sum_{j, k}^{\infty}\left(f_{k \sigma}-f_{j \sigma}\right) \frac{\psi_{k \sigma}^{*}(\mathbf{r}) \psi_{j \sigma}(\mathbf{r}) \psi_{j \sigma}^{*}\left(\mathbf{r}^{\prime}\right) \psi_{k \sigma}\left(\mathbf{r}^{\prime}\right)}{\varepsilon_{k \sigma}-\varepsilon_{j \sigma}+\omega+i \eta}
$$

is diagonal in the spins. Let us now expand the density response as

$$
n_{1 \sigma}(\mathbf{r}, \omega)=\sum_{n=-N}^{N} n_{1 n \sigma}(r, \omega) e^{i n \varphi}
$$

where in practice of course $N \leqslant L$. Inserting the form (5) of the Kohn-Sham orbitals, the response function is set up in the following way:

$$
\chi_{\sigma}\left(\mathbf{r}, \mathbf{r}^{\prime}, \omega\right)=\sum_{l, l^{\prime}=-N}^{N} \chi_{l l^{\prime} \sigma}\left(r, r^{\prime}, \omega\right) e^{i l \varphi} e^{-i l^{\prime} \varphi^{\prime}},
$$

where we define

$$
\chi_{l l^{\prime} \sigma}\left(r, r^{\prime}, \omega\right)=\sum_{j, k}^{\infty}\left(f_{k \sigma}-f_{j \sigma}\right) \frac{\Phi_{j k l}(r) \Phi_{j k l^{\prime}}\left(r^{\prime}\right)}{\varepsilon_{k \sigma}-\varepsilon_{j \sigma}+\omega+i \eta},
$$

with

$$
\Phi_{j k L}(r)=\sum_{\substack{l, l^{\prime} \\ l^{\prime}-l=L}} \phi_{j l}(r) \phi_{k l^{\prime}}(r) .
$$

For the xc kernel we use the adiabatic local-density approximation (ALDA)

$$
\begin{aligned}
f_{\mathrm{xc} \sigma \tau}\left(\mathbf{r}^{\prime}, \mathbf{r}^{\prime \prime}, \omega\right) & =\left.\frac{d^{2} e_{\mathrm{xc}}}{d n_{\sigma} d n_{\tau}}\right|_{n_{0}\left(\mathbf{r}^{\prime}\right)} \delta\left(\mathbf{r}^{\prime}-\mathbf{r}^{\prime \prime}\right) \\
& =\delta\left(\mathbf{r}^{\prime}-\mathbf{r}^{\prime \prime}\right) \sum_{m^{\prime}=-N}^{N} f_{\mathrm{xc}, m^{\prime} \sigma \tau}\left(r^{\prime}\right) e^{i m^{\prime} \varphi^{\prime}},
\end{aligned}
$$

where $e_{\mathrm{xc}}$ is the xc energy density of the homogeneous twodimensional electron gas, ${ }^{15}$ and

$$
f_{\mathrm{xc}, m^{\prime} \sigma \tau}\left(r^{\prime}\right)=\left.\frac{1}{2 \pi} \int_{0}^{2 \pi} d \varphi^{\prime} \frac{d^{2} e_{\mathrm{xc}}}{d n_{\sigma} d n_{\tau}}\right|_{n_{0}\left(\mathbf{r}^{\prime}\right)} e^{-i m^{\prime} \varphi^{\prime}} .
$$

In the ALDA, the xc kernel is frequency independent and real (for a recent discussion of alternative expressions for $f_{\text {xc }}$, see Ref. 16). The imaginary part of the Kohn-Sham response function (17) thus has to be put in by hand. In the following, we choose a value of $\eta=0.1 \mathrm{meV}$, corresponding to about $0.1-1 \%$ of the excitation energies under study.

Inserting everything into the response equation (16), we obtain

$$
\begin{aligned}
n_{1 l \sigma}(r, \omega)= & \int_{0}^{\infty} d r^{\prime} r^{\prime} \sum_{l^{\prime}=-N}^{N} \chi_{l l^{\prime} \sigma}\left(r, r^{\prime}, \omega\right) \mathrm{v}_{1 l^{\prime} \sigma}\left(r^{\prime}, \omega\right) \\
& +4 \pi^{2} e^{* 2} \sum_{\tau} \int_{0}^{\infty} d r^{\prime} r^{\prime} \int_{0}^{\infty} d r^{\prime \prime} r^{\prime \prime} \\
& \times \sum_{l^{\prime}=-N}^{N} \chi_{l l^{\prime} \sigma}\left(r, r^{\prime}, \omega\right) n_{1 l^{\prime} \tau}\left(r^{\prime \prime}, \omega\right) I_{l^{\prime}}\left(r^{\prime}, r^{\prime \prime}\right) \\
& +2 \pi \sum_{\tau} \int_{0}^{\infty} d r^{\prime} r^{\prime} \sum_{l^{\prime}, m=-N}^{N} \chi_{l l^{\prime} \sigma}\left(r, r^{\prime}, \omega\right) \\
& \times n_{1 m \tau}\left(r^{\prime}, \omega\right) f_{\mathrm{xc},\left(l^{\prime}-m\right) \sigma \tau}\left(r^{\prime}\right)
\end{aligned}
$$

Solving for the density response $n_{1}(\mathbf{r}, \omega)=n_{1 \uparrow}(\mathbf{r}, \omega)$ $+n_{1 \downarrow}(\mathbf{r}, \omega)$ for a given value of $\omega$ thus requires inversion of a complex matrix of dimension $2 N_{\text {grid }}(2 N+1)=1400$ (for $N=3$ and $N_{\text {grid }}=100$ ), which poses no problem in practice.

\section{External potential}

Let us now turn to the specific form of the bare confining potential of the quantum dot, $\mathrm{v}_{\mathrm{dot}}(r, \varphi)$, used to construct the electronic ground state in Sec. II A. First of all, we restrict ourselves to considering only potentials that have inversion symmetry. In other words, expansion $(8)$ of $\mathrm{v}_{\mathrm{dot}}(r, \varphi)$ con- 
tains only angular components $\mathrm{v}_{\mathrm{dot}, l}(r)$ with even $l$. We can then replace $e^{i l \varphi}$ in Eq. (9) by $\cos (l \varphi)$. Furthermore, to reduce the computational effort we shall restrict the values of $l$ to $l=0, \pm 2$, and \pm 4 .

We first consider the circularly symmetric part of the confining potential, $\mathrm{v}_{\text {dot, },}(r)$. This component describes the degree of nonparabolicity of our quantum dot. Pfannkuche and Gerhards ${ }^{6}$ assumed a form

$$
\mathrm{v}_{\mathrm{dot}, 0}(r)=\frac{m^{*}}{2}\left(\omega_{0}^{2} r^{2}+\omega_{1}^{2} r^{4}\right)
$$

where $\omega_{1} \ll \omega_{0}$. A different approach was chosen by Broido et $a l .^{3}$ : they constructed $\mathrm{v}_{\mathrm{dot}, 0}(r)$ as the electrostatic potential associated with a two-dimensional jellium disk of radius $R$ and uniform positive areal charge density $n_{+}$. Their result was

$$
\begin{aligned}
& \mathrm{v}_{\mathrm{dot}, 0}(r)=\mathrm{v}_{0}-4 e^{* 2} n_{+} R E\left(\frac{r}{R}\right), \quad r<R, \\
& \mathrm{v}_{\text {dot }, 0}(r)=\mathrm{v}_{0}-4 e^{* 2} n_{+} r\left[E\left(\frac{R}{r}\right)-\left(1-\frac{R^{2}}{r^{2}}\right) K\left(\frac{R}{r}\right)\right], \quad r>R,
\end{aligned}
$$

where $\mathrm{v}_{0}=2 \pi e^{* 2} n_{+} R$. Here $K$ and $E$ denote complete elliptic integrals of the first and second kinds. It is easy to see that Eq. (25) is the small-r expansion of Eq. (26), identifying the coefficients as

$$
\begin{gathered}
\omega_{0}^{2}=\frac{\pi e^{* 2} n_{+}}{m^{*} R}, \\
\omega_{1}^{2}=\frac{3 \pi e^{* 2} n_{+}}{16 m^{*} R^{3}} .
\end{gathered}
$$

The main difference between the two forms of $\mathrm{v}_{\mathrm{dot}, 0}(r)$ is that (25) grows as $r^{4}$ for large $r$, whereas (26) approaches the constant $\mathrm{v}_{0}$ as $1 / r$. This difference is of less importance for the electronic ground state, since the two potentials are very similar in the interior region of the dot where the electronic density is concentrated, but it can be expected to substantially affect the electronic excitations.

We now turn to the components of $v_{\text {dot }}$ that break the circular symmetry. In Refs. 4 and 6 this is accomplished by including terms of square symmetry, i.e., proportional to $x^{2} y^{2}$. Similarly, one can add on terms proportional to $x^{2}$ or $y^{2}$, describing elliptic elongation of the dot along the $x$ or $y$ axis. ${ }^{8}$ In this manner, one arrives at

$$
\begin{aligned}
& \mathrm{v}_{\mathrm{dot}, \pm 2}(r)=a r^{2} \\
& \mathrm{~V}_{\mathrm{dot}, \pm 4}(r)=b r^{4},
\end{aligned}
$$

which introduces two more adjustable parameters $a$ and $b$, in addition to $\omega_{0}$ and $\omega_{1}$.

Again, an alternative approach to the construction of $\mathrm{v}_{\mathrm{dot}}(r, \varphi)$ is to start out with a flat jellium disk of uniform positive charge $n_{+}$, but this time with a noncircular shape parametrized as $R(\varphi)$. Once a particular form for $R(\varphi)$ has been chosen, the associated electrostatic potential is calculated as

$$
\begin{aligned}
\mathrm{V}_{\mathrm{dot}}(r, \varphi)= & \tilde{\mathrm{V}}_{0}-e^{* 2} n_{+} \int_{0}^{2 \pi} d \varphi^{\prime} \int_{0}^{R\left(\varphi^{\prime}\right)} \\
& \times \frac{r^{\prime} d r^{\prime}}{\sqrt{r^{2}+r^{\prime 2}-2 r r^{\prime} \cos \left(\varphi-\varphi^{\prime}\right)}},
\end{aligned}
$$

where now

$$
\tilde{\mathrm{v}}_{0}=e^{* 2} n_{+} \int_{0}^{2 \pi} d \varphi^{\prime} R\left(\varphi^{\prime}\right) .
$$

The $r^{\prime}$ integral in Eq. (29) can be performed analytically, and the remaining integration over $\varphi^{\prime}$ has to be done numerically for general $R\left(\varphi^{\prime}\right)$. If $R\left(\varphi^{\prime}\right)=$ const, one recovers the previous result [Eqs. (26)]. The next step is then to construct the angular components $\mathrm{V}_{\mathrm{dot}, l}(r)$ using Eq. (9), which in general requires a second straightforward numerical integration. be

The large- $r$ behavior of the dot potential (29) is found to

$$
\mathrm{V}_{\mathrm{dot}}(r \rightarrow \infty, \varphi)=\widetilde{\mathrm{v}}_{0}-\frac{e^{* 2} n_{+}}{2 r} \int_{0}^{2 \pi} d \varphi^{\prime} R\left(\varphi^{\prime}\right)^{2}
$$

independent of $\varphi$. This means that for large distances only the $l=0$ component of $\mathrm{v}_{\mathrm{dot}}(r, \varphi)$ survives (approaching the constant $\widetilde{\mathrm{v}}_{0}$ ), and the higher- $l$ components go to zero. This is again in contrast with the form (28) for the noncircular components of the dot potential, which (unphysically) keep increasing with distance. As noted before in the case of the circular dot, this difference is not expected to have a large impact on the electronic ground state, but it may become important for higher excitations. We therefore conclude that in general it is preferable to work with dot potentials constructed according to Eq. (29), thus avoiding effects caused by an unphysical behavior in the large- $r$ region.

\section{RESULTS AND DISCUSSION}

The experiments presented in Refs. 9 and 10 were performed on self-assembled InAs quantum dots embedded into GaAs. The diameter of the dots is estimated to be about 20 $\mathrm{nm}$, and the height to be about $7 \mathrm{~nm}$. As outlined above, we treat the quantum dots as two-dimensional systems. Within our model, we also ignore the presence of the wetting layer.

From their measurements, the authors of Refs. 9 and 10 deduced an effective mass $m^{*}=0.08 m_{e}$ (where $m_{e}$ is the bare electronic mass). Furthermore, we take the effective charge as $e^{*}=e / \sqrt{\varepsilon}$ ( $e$ is the bare electronic charge), using $\varepsilon=15.15$ for the dielectric constant, i.e., the bulk value of InAs, and we employ an effective $g$-factor $g *=-0.44$. For the curvature of the bare confining potential of the dot close to its center, we take a value of $\omega_{0}=45 \mathrm{meV}$, which leads to $n_{+}=0.7 \times 10^{15} \mathrm{~cm}^{-2}$ via relation (27) (for $R=100 \AA$ ).

The specific form of the bare confining potential of the dot can now be constructed using one of the two approaches discussed in Sec. II C. In the following, our choice is to construct $\mathrm{V}_{\mathrm{dot}}(r, \varphi)$ as the electrostatic potential associated 


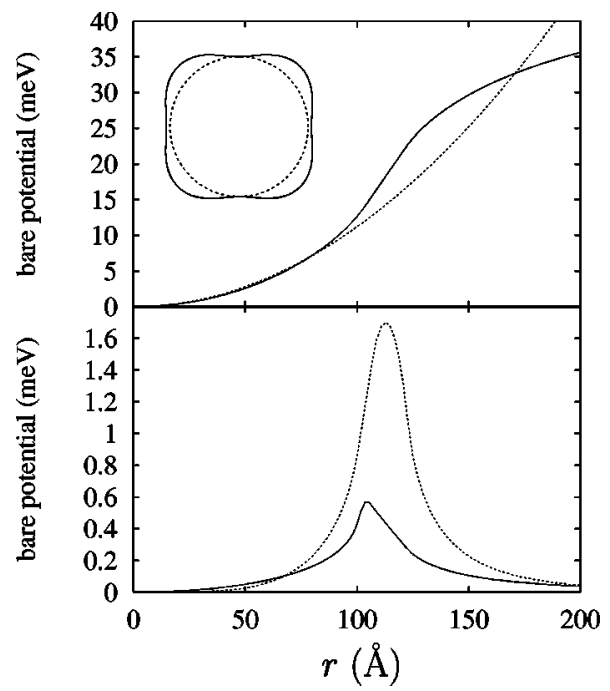

FIG. 1. Angular components $\mathrm{v}_{\mathrm{dot}, l}(r)$ of the bare confining potential of a quantum disk parametrized by Eq. (32). The shape of the disk is shown in the inset, together with a circle of radius $R_{0}$ $=100 \AA$. Top: $l=0$ component (full line). For comparison, the dashed line shows a parabolic potential with the same curvature in the interior region. Bottom: $l=2$ (full line) and $l=4$ (dashed line) components.

with a disk whose radius is parametrized as

$$
R(\varphi)=R_{0}+R_{2} \sin ^{2} \varphi+R_{4} \sin ^{2} \varphi \cos ^{2} \varphi .
$$

For $R_{0}$, we take the estimated radius of the dots, i.e., $R_{0}$ $=100 \AA$. The parameter $R_{2}$ indicates an elliptic elongation of the quantum dot along the $y$ axis. We adopt a value of $R_{2}=5 \AA$, as estimated in Ref. 9. For the parameter $R_{4}$ that causes an anisotropy of square symmetry, no direct experimental numbers are available. In the following we choose $R_{4}=83 \AA$, so that the value of $R_{4} \sin ^{2} \varphi \cos ^{2} \varphi$ is at most $20 \%$ of $R_{0}$. The resulting shape of the dot, a rectangle with rounded-off corners, is shown as inset in Fig. 1. For comparison, a circle with radius $R_{0}$ is also indicated.

In Fig. 1 we plot the angular components $\mathrm{v}_{\mathrm{dot}, l}(r)$ of the bare confining potential of the quantum disk parametrized by Eq. (32). The top part shows the circularly symmetric part $\mathrm{V}_{\mathrm{dot}, 0}(r)$, together with a parabolic potential that would correspond to the case $\mathrm{v}_{\mathrm{dot}}(r)=m^{*} \omega_{0}^{2} r^{2} / 2$. The bottom part shows the $l=2$ and 4 components. Note that if the elongation of the dot is along the $x$ axis [replace $R_{2} \sin ^{2} \varphi$ by $R_{2} \cos ^{2} \varphi$ in Eq. (32)], then $\mathrm{v}_{\text {dot, } 2}(r)$ changes sign. From Fig. 1 it is evident that the deviation from circular symmetry affects the confining potential mainly in the region around the edge of the dot, whereas the inner region of the dot sees a nearly parabolic potential.

Let us now discuss our main numerical results. Figure 2 shows the calculated peak positions of the photoabsorption cross section $\sigma(\omega)$ versus applied magnetic field, for a quantum dot with $N=2$ electrons. Here and in the following, we assume the quantum dots to be elongated along the $x$ axis. We then calculate the photoabsorption spectra for two different polarizations of the FIR radiation, in $x$ and $y$ directions, respectively. The symbols in Fig. 2 denote the average of the two spectra. For comparison, the full lines show the expected behavior of $\omega_{ \pm}$according to Eq. (1), with a fitted value of

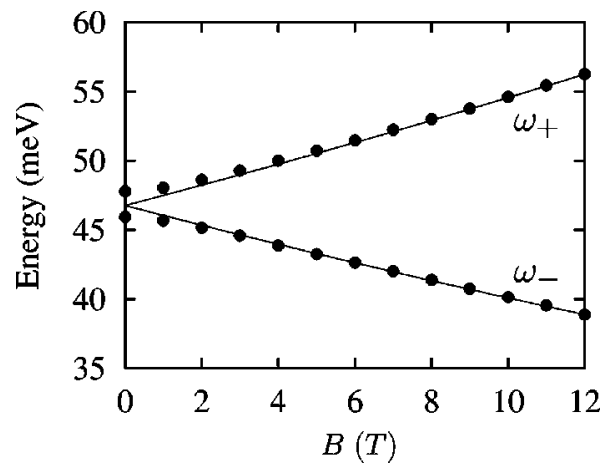

FIG. 2. Calculated peak positions of the photoabsorption spectrum for a noncircular, nonparabolic quantum dot with $N=2$ electrons. The full lines show the results one would obtain for a circular parabolic dot; see Eq. (1).

$\omega_{0}=46.8 \mathrm{meV}$. We see that for higher magnetic fields, the calculated peaks follow the simple law of Eq. (1). For small $B$, however, some deviations occur, and a splitting of about 2 meV remains even for $B=0 T$, in accordance with observation. ${ }^{9,10}$

For $N=2$, the quantum dot contains a full $s$ shell, ${ }^{17}$ and the behavior is very similar to a parabolic dot. As soon as the $p$ shell is occupied, however, deviations from the parabolic case become much more pronounced. In Fig. 3 we plot the

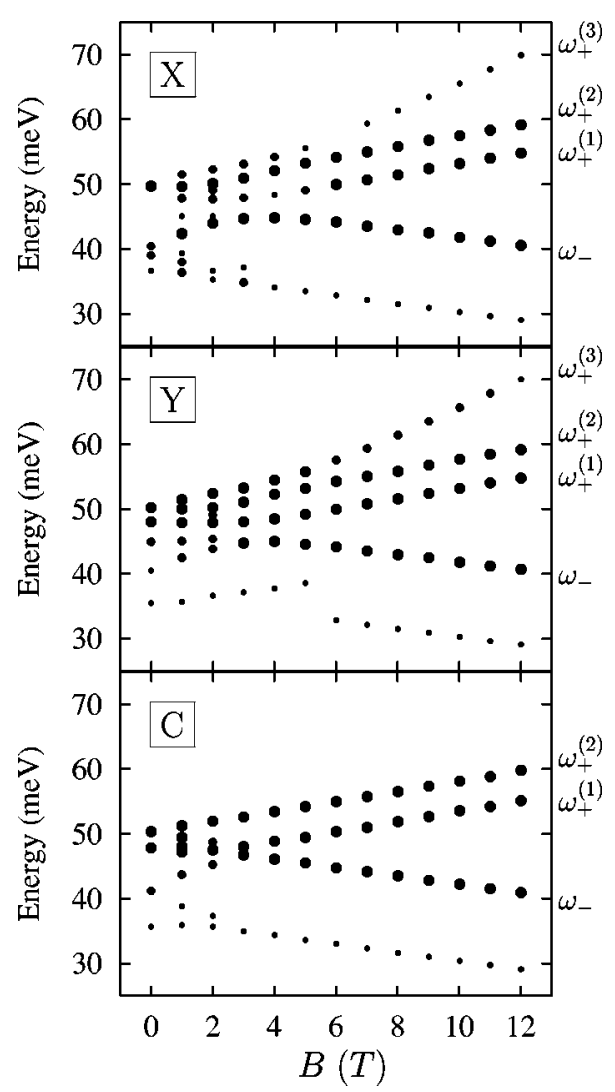

FIG. 3. Calculated peak positions of the photoabsorption spectrum for a quantum dot with $N=3$ electrons. Bottom: nonparabolic but circular dot. Top and middle: nonparabolic, noncircular dot, with polarization of the FIR radiation parallel $(X)$ and perpendicular $(Y)$ to the direction of elongation. The $\omega_{+}$mode splits up into three subpeaks $\omega_{+}^{(1)}, \omega_{+}^{(2)}$, and $\omega_{+}^{(3)}$, as indicated. 


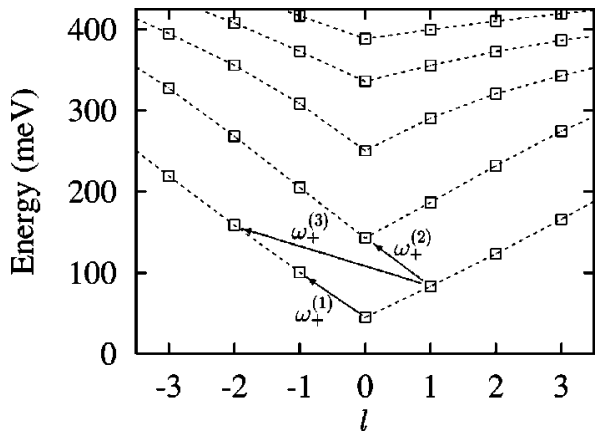

FIG. 4. Energy levels of a single electron in the bare potential of the quantum dot from Fig. 1 at $B=12 T$. The levels are drawn vs their angular momentum quantum number $l$, and dashed lines connect levels with the same principal quantum number $n$. The arrows indicate the single-particle transitions that constitute the threefold splitting of $\omega_{+}$.

FIR peak positions for an $N=3$ quantum dot versus $B$. The intensities of the absorption peaks are approximately indicated by the size of the symbols. The two top figures show results for the noncircular dot, for two different polarizations of the FIR radiation: parallel to the direction of elongation of the dot (the $x$ axis) and perpendicular to it. In both cases we find very rich spectra. For small magnetic fields, there are substantial differences between the two polarizations, for $B>6 T$ these differences disappear.

The bottom part of Fig. 3 shows the spectra for a circular (but still nonparabolic) dot. These results have been obtained by setting $\mathrm{v}_{\mathrm{dot}, l}(r)=0$ for $l= \pm 2$ and \pm 4 , but using the same $\mathrm{v}_{\mathrm{dot}, 0}(r)$ as in the two figures above. By comparison between the lower and the two upper parts of Fig. 3, we can now clearly distinguish between those effects related to breaking the circular symmetry and those caused by nonparabolicity. The latter leads to a splitting of the $\omega_{+}$mode into two almost equally strong subpeaks, $\omega_{+}^{(1)}$ and $\omega_{+}^{(2)}$, separated by about 5 $\mathrm{meV}$, plus the appearance of a weaker signal connected with the $\omega_{-}$mode and approximately $10 \mathrm{meV}$ below it. We mention that similar results have been previously obtained by Hawrylak and co-workers. ${ }^{2,18}$

By introducing a noncircular anisotropy, the $\omega_{+}$mode acquires a third subpeak $\omega_{+}^{(3)}$, in agreement with the experimentally observed behavior (see Fig. 3 of Ref. 10). We find that the evolution of this signal with magnetic field is different from that of the two other subpeaks of $\omega_{+}$: it becomes weaker with increasing $B$, and its separation from $\omega_{+}^{(1)}$ and $\omega_{+}^{(2)}$ is growing.

To understand the origin of the threefold splitting of $\omega_{+}$, it is helpful to resort to a simple single-particle picture. Figure 4 shows the energy levels of a single electron confined in the bare potential of our quantum dot at $B=12 T$, versus angular momentum quantum number $l$ (see Ref. 17). The dashed lines connect energy levels to which one can assign the same principal quantum number $n$. The distribution of energy levels shown in Fig. 4 is very similar to that for a parabolic dot, which is governed by the formula

$$
E_{n l}=(2 n+|l|+1) \sqrt{\omega_{0}^{2}+\frac{\omega_{c}^{2}}{4}}-\frac{\omega_{c} l}{2}
$$

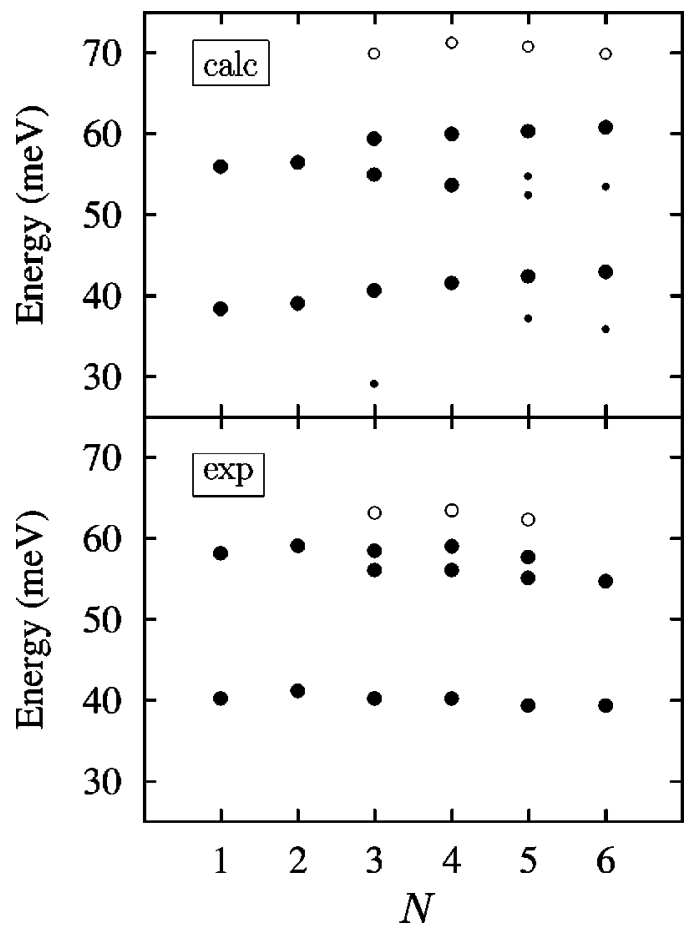

FIG. 5. Comparison between the calculated and experimental peak positions in the photoabsorption spectra for quantum dots with $1 \leqslant N \leqslant 6$ electrons at $B=12 T$. The open circles indicate those signals that we find to be related to a broken circular symmetry of the dots.

(see the discussion in the review article by Ashoori ${ }^{1}$ ). In contrast with the strictly parabolic case, the vertical distances between the levels in Fig. 4 are not constant.

In this simple picture (which remains qualitatively valid for $N>1$ even if the electron-electron interaction is included), the subpeaks of $\omega_{+}$can be identified with singleparticle transitions. For circular symmetry, these are governed by the selection rule for dipole transitions, $\Delta l= \pm 1$. In this simplified scenario, $\omega_{+}^{(1)}$ and $\omega_{+}^{(2)}$ arise from $1 s \rightarrow 1 p_{-}$ and $1 p_{+} \rightarrow 2 s$ transitions, as indicated in Fig. 4. Breaking the circular symmetry means that the selection rule can be violated: we find that the $\omega_{+}^{(3)}$ mode originates from the transition $1 p_{+} \rightarrow 1 d_{-}$, i.e., $\Delta l=-3$. The oscillator strength of this mode is of course directly related to the degree of anisotropy, which in our case is only small.

We have found that the position of $\omega_{+}^{(3)}$ with respect to $\omega_{+}^{(1)}$ and $\omega_{+}^{(2)}$ is insensitive to small variations in the choice of the parameters $R_{2}$ and $R_{4}$ in Eq. (32). A weak signal at $\omega_{+}^{(3)}$ will be present even if only one of the two is nonzero. However, the value of $R_{2}=5 \AA$ is more or less dictated by the experimentally observed splitting between $\omega_{+}$and $\omega_{-}$ for $N=2$ at zero magnetic field, see above. One then observes that choosing a finite value for $R_{4}$ increases the intensity of the third subpeak.

The central result of this work is presented in Fig. 5. It shows a comparison between the calculated and measured ${ }^{9}$ peak positions in the photoabsorption spectra for quantum dots with $1 \leqslant N \leqslant 6$ electrons ${ }^{19}$ at $B=12 T$. We find that the main experimental features are reproduced by the calculation. For $N=1$ and 2, the system behaves very similarly to a circular parabolic dot, as noted before, i.e., there are only 
two signals at $\omega_{+}$and $\omega_{-}$. As soon as the $p$ shell becomes occupied, i.e., for $N \geqslant 3$, the $\omega_{+}$mode splits up. As explained above, the $\omega_{+}^{(3)}$ signal (indicated here by the open circles) is related to a breaking of the circular symmetry.

We note that the calculation yields a splitting between the three subpeaks of $\omega_{+}$that is greater than the one found in the experiment. Also, for $N=6$ the experiment yields only a single signal at $\omega_{+}$. These differences between theory and experiment are to be attributed to the simplified nature of our model that treats the self-assembled dots as two-dimensional disks. In particular, the observed differences at $N=6$ are most likely due to our neglecting the presence of the wetting layer.

The main effect of the wetting layer is to introduce a continuum of states above a certain energy threshold, ${ }^{2}$ limiting the number of bound states localized in the selfassembled dot. As the number of electrons grows, the energy levels are shifted toward higher energies due to the increasing interaction energy, and more and more states are pushed into the continuum, up to a point where no additional electron can be bound. From the absence of $\omega_{+}^{(2)}$ and $\omega_{+}^{(3)}$ for $N=6$ observed in the experiment, we infer that the relevant states involved in the transitions $\left(2 s\right.$ and $\left.1 d_{-}\right)$would in reality fall into the wetting layer continuum and the transitions would lose most of their strength. However, for $N<6$ these states must still be located in the discrete part of the energy spectrum, since the associated transitions are present in the experiment.

This effect was accounted for in Ref. 18 by using a truncated basis of only few bound states in the numerical diagonalization, and it was indeed found that for $N=6$ there is only a single peak at $\omega_{+}^{(1)}$. However, the calculations in Ref. 18 assumed circular symmetry and produced at most a twofold splitting of $\omega_{+}$for $N=3,4$, and 5, in contrast with experiment. We therefore conclude that, in spite of the deviations from experiment mentioned above, our calculations clearly establish that the presence of $\omega_{+}^{(3)}$ for $N=3,4$, and 5 is due to a breaking of circular symmetry of the quantum dots.

\section{CONCLUSION}

In this work, we developed a theoretical description of collective charge-density excitations of noncircular quantum dots in a magnetic field. The computational scheme presented here allows one to obtain information on the geometry of quantum dots from their electronic response properties.

Our specific aim was to reproduce and explain recent experimental results, obtained for self-assembled InAs quantum dots. In these FIR photoabsorption measurements, one detects a threefold splitting of the upper branch $\omega_{+}$of the collective charge-density mode in a magnetic field, depending on the number of electrons present in the dot. Our study has shown that these experimental features are closely related to the shape of the dots: in addition to nonparabolicity (due to the finite dot radius), it is essential to account for anisotropy effects, leading to a noncircular confining potential. To our knowledge, here we have presented the first fully self-consistent spin-density-functional calculations of ground state plus linear response for anisotropic quantum dots with up to six electrons.

With our calculations we were able to reproduce and explain the main features of the FIR spectroscopy measurements conducted in Refs. 9 and 10. However, the agreement was not fully quantitative. This may be attributed to the simplicity of our model, which treats self-assembled, lensshaped (with a possible elliptic or pyramidal distortion) quantum dots sitting on a wetting layer as two-dimensional quantum disks. It may safely be expected that this model leads to much better quantitative results for quantum-dot devices that are produced by mesa-etching techniques, also known as vertical quantum dots. ${ }^{20}$

\section{ACKNOWLEDGMENTS}

This work was supported by Research Board Grant RB 96-071 from the University of Missouri and by NSF Grant No. DMR-9706788. We thank Axel Lorke for stimulating discussions.
${ }^{1}$ R. C. Ashoori, Nature (London) 379, 413 (1996).

${ }^{2}$ L. Jacak, P. Hawrylak, and A. Wójs, Quantum Dots (Springer, Berlin, 1998).

${ }^{3}$ D. A. Broido, K. Kempa, and P. Bakshi, Phys. Rev. B 42, 11400 (1990).

${ }^{4}$ V. Gudmundsson and R. R. Gerhards, Phys. Rev. B 43, 12098 (1991).

${ }^{5}$ T. Demel, D. Heitmann, P. Grambow, and K. Ploog, Phys. Rev. Lett. 64, 788 (1990).

${ }^{6}$ D. Pfannkuche and R. R. Gerhards, Phys. Rev. B 44, 13132 (1991).

${ }^{7}$ Z. L. Ye and E. Zaremba, Phys. Rev. B 50, 17217 (1994).

${ }^{8}$ K. Hirose and N. S. Wingreen, Phys. Rev. B 59, 4604 (1999).

${ }^{9}$ M. Fricke, A. Lorke, J. P. Kotthaus, G. Medeiros-Ribeiro, and P. M. Petroff, Europhys. Lett. 36, 197 (1996).

${ }^{10}$ A. Lorke, M. Fricke, B. T. Miller, M. Haslinger, J. P. Kotthaus, G. Medeiros-Ribeiro, and P. M. Petroff, in Compound Semicon- ductors 1996, edited by M. S. Shur and R. A. Suris, IOP Conf. Proc. No. 155 (Institute of Physics, Bristol, 1997), p. 803.

${ }^{11}$ B. T. Miller, W. Hansen, S. Manus, R. J. Luyken, A. Lorke, J. P. Kotthaus, S. Huant, G. Medeiros-Ribeiro, and P. M. Petroff, Phys. Rev. B 56, 6764 (1997); R. J. Luyken, A. Lorke, M. Haslinger, B. T. Miller, M. Fricke, J. P. Kotthaus, G. MedeirosRibeiro, and P. M. Petroff, Physica E 2, 704 (1998); A. Lorke and R. J. Luyken, Physica B 256-258, 424 (1998).

${ }^{12}$ G. Vignale and M. Rasolt, Phys. Rev. Lett. 59, 2360 (1987); Phys. Rev. B 37, 10685 (1988).

${ }^{13}$ M. Ferconi and G. Vignale, Phys. Rev. B 50, 14722 (1994).

${ }^{14}$ E. Lipparini, N. Barberán, M. Barranco, M. Pi, and Ll. Serra, Phys. Rev. B 56, 12375 (1997); E. Lipparini and Ll. Serra, ibid. 57, R6830 (1998); M. Pi, M. Barranco, A. Emperador, E. Lipparini, and Ll. Serra, ibid. 57, 14783 (1998); Ll. Serra, M. Barranco, A. Emperador, M. Pi, and E. Lipparini, ibid. 59, 15 290 (1999) 
${ }^{15}$ B. Tanatar and D. M. Ceperley, Phys. Rev. B 39, 5005 (1989).

${ }^{16}$ C. A. Ullrich and G. Vignale, Phys. Rev. B 58, 15756 (1998).

${ }^{17}$ For a system with broken circular symmetry, the angular momentum is no longer a good quantum number, and the electronic orbitals become linear combinations of pure $l$ states, see Eq. (5). However, if the anisotropy of the dots is viewed as a small perturbation, one may continue to speak of $s, p, d, \ldots$ electrons, since for each $\psi_{j \sigma}$ expansion (5) is then dominated by a single term with well-defined $l$ (we use the standard notation s for $l$ $=0, \quad p_{ \pm}$for $l= \pm 1$, etc. $)$.
${ }^{18}$ A. Wójs and P. Hawrylak, Phys. Rev. B 53, 10841 (1996).

${ }^{19}$ The calculations for $N=1$ have not been done within the localdensity approximation of density-functional theory, but simply by solving the Schrödinger equation for a single electron in the bare potential of the dot. In Eq. (16) the electron then responds to the external potential $\mathrm{v}_{1}(\mathbf{r}, \omega)$ only.

${ }^{20}$ S. Tarucha, D. G. Austing, T. Honda, R. J. van der Hage, and L. P. Kouwenhoven, Phys. Rev. Lett. 77, 3613 (1996); T. H. Oosterkamp, J. W. Janssen, L. P. Kouwenhoven, D. G. Austing, T. Honda, and S. Tarucha, ibid. 82, 2931 (1999). 